trenchantly as he did twenty years ago and the theory emerges from the trial without much of a stain on its character. The second charge concerns the length-tension relationship, one of the corner-stones of the crossbridge hypothesis. Gordon, A.F. Huxley and Julian showed in 1966 that the tension developed by a muscle fibre depends linearly on the overlap between the filament, though if stimulation of stretched fibres is prolonged tension continues to rise slowly. This was ascribed to the shortening of some of the sarcomeres. Pollack states that he does not observe such a shortening, at least using light diffraction. On the other hand Julian says he does, using photography. A fair verdict would be that the charge is "not proven."

Ingels makes what seems to be his second contribution to the field with a chapter on "Unifying Concepts" which attempts to unify his first contribution, a non- crossbridge theory, to crossbridge theory, but little else. As organiser of the conference he must bear some responsibility for the fact that nearly two-thirds of the book is unrepresentative of current research in the field. The chapters on crossbridge theory and practice might serve as an introduction to the field and contain some new results particularly from $\mathrm{X}$-ray diffraction studies, but they are too brief. The discussion never gets away from the two issues reported above. The question of whether current results from structural, biochemical and mechanical studies can be explained on other than a crossbridge model is not raised. There is an Appendix from Ingels with a chronology of muscle theories, some of which are splendidly batty.

R.M. Simmons is Lecturer in Physiology at University College, London, UK.

\section{Molecular basis of tumourigenicity}

\section{Dennis D. Cunningham}

Surfaces of Normal and Malignant Cells. Edited By R.O. Hynes. Pp.471. (Wiley: Chichester, UK, and New York, 1979.) £24.

THE cell surface has become a prime target for studies on the molecular basis of tumourigenicity. In a lucid introductory chapter, the editor summarises reasons for this, discusses issues basic to such studies (for example, do properties of transformed cells in culture accurately reflect the properties of malignant cells in vivo?), and sets the stage for the subsequent contributions.

A chapter on morphology of normal and transformed cells is followed by three chapters on cell surface composition which emphasise glycolipids, proteins and glycoproteins, and mucopolysaccharides. Two chapters focus on enzymes of normal and transformed cells, and there is a chapter on immune responses to C-type virus-induced tumours. From here on, the chapters mostly deal with control processes in normal cells: early events in growth stimulation, surface transport components of erythrocytes, adhesive specificity among embryonic cells, and the cell surface and development in the cellular slime mould Dictyostelium discoideum.

A theme which recurs in this volume is that the several properties which appear to correlate generally with the transformed state are not invariably found in all transformed or tumourigenic cells; it is probably certain combinations of these, along with alterations yet to be defined, that collectively underlie malignancy.
Given present uncertainties about which of the many alterations found in transformed cells and their surfaces might be causally related to malignancy, and the fact that any given topic on regulation of cellular activities could relate to alterations basic to malignancy, the editor faced a real challenge when choosing the issues to be addressed in this volume.

Although most of the choices were excellent, it would have been an easy matter to justify individual chapters on assays for tumourigenicity (malignancy?) of cultured cells, and the use of melanoma lines with specific metastatic potentials to probe cell surface properties related to metastasis. As emphasised by the editor, the commonly

\section{Wave functions for atoms and molecules}

\section{A.D. Buckingham}

The Calculation of Molecular Orbitals. By J.C. Slater. Pp. 104. (Wiley: New York and Chichester, UK, 1979.) £11.50; \$23.

PROFESSOR J.C. Slater was a theoretician of the first rank and a prodigious writer of textbooks. The present work is the last in a series of eight books by Slater on the quantum theory of matter (the first was published in 1960). The manuscript of this book was found among his papers following his death in 1976, the only addition being a list of a small number of references.

In the last ten years of his life, Slater was concerned with the local-density form of the self-consistent field; his work led to the so-called $X \alpha$ method, and he and many used assays for tumourigenicity of cultured cells depend on growth and survival of the cells and not generally on their ability to invade and metastasise, even though the latter two properties distinguish malignant tumours from ones that are benign.

As "a mere collection of data would soon become out of date . . . the authors were encouraged to extrapolate from the established facts and discuss hypotheses concerning their significance"'. As a result, the individual chapters are generally conceptual in nature, and provide the reader with interpretations of results. Thus, the volume does what this type of book should: synthesise and extrapolate rather than present previously published data. At the same time, each chapter is quite well referenced, providing the reader with ready access to the original literature. In general, the individual chapters are well written and the authors emphasise questions that remain to be answered. Two chapters, "Surface Membrane Enzymes in Neoplasia" and "The Cell Surface and Development in the Cellular Slime Mould Dictyostelium discoideum', were disappointing. Both were rather long on facts and short on interpretation. Unfortunately, the latter chapter did not contain a much needed summary section.

My overall impression of this book is quite favourable. It generally emphasises ideas and questions; at the same time, it contains enough experimental facts to be informative and credible. With exceptions in only several parts, the book is well integrated, a feature which should increase its general appeal.

Dennis D. Cunningham is Professor of Microbiology at the University of California at Irvine.

others applied it to a wide range of problems in theoretical physics and chemistry.

This new book of only a hundred pages centres on the SCF $X \alpha$ method for obtaining approximate electronic wavefunctions for atoms and molecules.

Applications of the $X \alpha$ method have involved approximations which impose limitations on the validity of the results. Slater's purpose in writing this book was to suggest a different scheme for solving the SCF equations. He turned to the cellular method, developed by Wigner and Seitz for handling the problem of computing the orbitals of a crystal. (Slater himself was a pioneer in this energy-band theory.)

All those who are interested in the SCF $X \alpha$ method will find this a most useful and informative book. It is the writing of a mature scientist, one of the masters of theoretical physics and chemistry.

A.D. Buckingham is Professor of Chemistry at the University of Cambridge, UK. 